\title{
LANDAU-KOLMOGOROV INEQUALITIES FOR SEMIGROUPS AND GROUPS
}

\author{
MELINDA W. CERTAIN AND THOMAS G. KURTZ
}

\begin{abstract}
An elementary functional analytic argument is given showing how inequalities of the form $\left\|f^{(k)}\right\|^{n} \leqslant K_{n, k}\|f\|^{n-k}\left\|f^{(n)}\right\|^{k}$, where $f$ is a real, $n$-times differentiable function and $\|\cdot\|$ denotes the sup norm on $(0, \infty)$ (or $(-\infty, \infty)$ ), yield corresponding inequalities, $\left|A^{k} x\right|^{n} \leq K_{n, k}|x|^{n-k}\left|A^{n} x\right|^{k}$, for generators of linear contraction semigroups (or groups) on arbitrary Banach spaces with norm $\mid \%$. Since Landau, Kolmogorov, Schoenberg and Cavaretta have established the function inequalities with the best possible constants, this argument gives the generator inequalities with the best possible constants for general Banach spaces extending work of Kallman and Rota, Hille and others. Questions concerning the best possible constants for specific Banach spaces remain open.
\end{abstract}

1. Introduction. In 1913 Edmund Landau [11] established that if the realvalued functions $f$ and $f^{\prime \prime}$ are bounded on $[0, \infty]$ then

$$
\left\|f^{\prime}\right\|^{2} \leqslant 4\|f\|\left\|f^{\prime \prime}\right\|
$$

where $\|\cdot\|$ denotes the sup norm. He showed that the constant 4 is best possible.

In 1967 R. Kallman and G.-C. Rota [9] proved what is now a well-known generalization of the above: If $A$ is the generator of a strongly continuous linear contraction semigroup and $x$ is in the domain of $A^{2}$ then

$$
|A x|^{2} \leqslant 4|x|\left|A^{2} x\right|
$$

where $|\cdot|$ denotes the norm in the Banach space on which the semigroup acts.

In this note we show that (2) may be obtained from (1) via elementary functional analysis. More generally, if $n$ is a positive integer, $k=1, \ldots$, $n-1$, and one has constants $M_{n, k}$ such that

$$
\left\|f^{(k)}\right\|^{n} \leqslant M_{n, k}\|f\|^{n-k}\left\|f^{(n)}\right\|^{k}
$$

for $f$ a real-valued function on $(0, \infty)$, then a functional analytic argument yields

Received by the editors April 6, 1976.

AMS (MOS) subject classifications (1970). Primary 47D05, 47D10; Secondary 47B44.

Key words and phrases. Semigroup generators, group generators, Kallman-Rota Inequality.

- American Mathematical Society 1977 


$$
\left|A^{k} x\right|^{n} \leqslant M_{n, k}|x|^{n-k}\left|A^{n} x\right|^{k}
$$

for $A$ the generator of a strongly continuous linear semigroup.

Since a 1970 paper by I. Schoenberg and A. Cavaretta [13] (see also [1] and [12]) establishes (3) and gives the best possible constants $M_{n, k}$, the theorems here establish (4) with the same constants; these constants are the best possible for (4) also since the semigroup of translations has differentiation as its generator.

In 1939 Kolmogorov [10] obtained constants $C_{n, k}$ such that if $f$ is a bounded real-valued function on $(-\infty, \infty)$ with bounded $n$th derivative then

$$
\left\|f^{(k)}\right\|^{n} \leqslant C_{n, k}^{n}\|f\|^{n-k}\left\|f^{(n)}\right\|^{k}
$$

and he showed his constants $C_{n, k}$ were best possible on $(-\infty, \infty)$. (Kolmogorov's constants are written to the $n$th power because $C_{n, k}^{n}$ is always rational.)

In the same way that (4) follows from (3), (5) implies that if $A$ generates a group of isometries then

$$
\left|A^{k} x\right|^{n} \leqslant C_{n, k}^{n}|x|^{n-k}\left|A^{n} x\right|^{k}
$$

for every $x$ in the domain of $A^{n}$.

Since the constants in (4) and (6) are the same as the constants in the classical function inequalities, these results improve the results of Hille [7] as well as some of those of Gindler and Goldstein [5]. For example, the best possible value for $M_{3,2}$ of equation (4) is 24 (see Schoenberg [12, p. 152]), whereas Gindler and Goldstein obtain 288; similarly, the best possible value for $M_{3,1}$ is $243 / 8$ which can be compared to $1575 / 4$ of Gindler and Goldstein.

Hille does not exhibit explicit values for his constants in the semigroup case except for $n=2, k=1$, for which his method yields 8 instead of 4 . As he says, his is essentially an existence proof and does not give the best constants in general. Hille does obtain the best constants in the group case for $n=2$ and $n=3$ but not for $n=4$; in particular, for $n=4, k=2$, Hille obtains $16 / 9$ whereas the Kolmogorov constant $C_{4,2}^{4}$ is $36 / 25$. Some indication as to why the cases $n \geqslant 4$ do not yield to Hille's method can be found in Schoenberg [12] and Certain [2].

The best constants over the class of all linear contraction semigroups (or groups) are thus established; however, questions of the best constants in particular cases (for example, semigroups of $L_{p}$-spaces or, even more particularly, the constants for a specified operator) remain. For results in this direction as well as additional references to earlier work see Gindler and Goldstein [5], Goldstein [6], Holbrook [8], and Trebels and Westphal [14].

Note. It has been brought to the attention of the authors that the basic idea of Theorem I is contained in a remark of Z. Ditzian added in proof to [3] and used by him in [4]. 
2. Statement and proofs of theorems. Denote by $X$ a real Banach space with

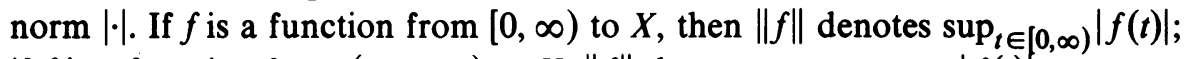
if $f$ is a function from $(-\infty, \infty)$ to $X,\|f\|$ denotes $\sup _{t \in(-\infty, \infty)}|f(t)|$.

The constants of Schoenberg and Cavaretta and those of Kolmogorov, as described in the introduction, will be denoted by $M_{n, k}$ and $C_{n, k}$, respectively, $n$ a positive integer, $k=1, \ldots, n-1$.

THEOREM I. Suppose $n$ is a positive integer. If $f$ is a function from $[0, \infty)$ to $X$ such that $f$ has $n$ continuous derivatives, and $\|f\|$ and $\left\|f^{(n)}\right\|$ are finite, then

$$
\left\|f^{(k)}\right\|^{n} \leqslant M_{n, k}\|f\|^{n-k}\left\|f^{(n)}\right\|^{k}, \quad k=1,2, \ldots, n-1 .
$$

Proof. Let $l$ be a linear functional on $X$ with $|l|_{X^{*}}=1$. Then $h(t)$ $=l(f(t))$ is real valued and $n$-times continuously differentiable with $h^{(k)}(t)$ $=l\left(f^{(k)}(t)\right)$.

Inequality (3) implies

$$
\begin{aligned}
\sup _{t \geqslant 0}\left|l\left(f^{(k)}(t)\right)\right| & \leqslant M_{n, k}\left(\sup _{t \geqslant 0}|l(f(t))|\right)^{n-k}\left(\sup _{t \geqslant 0}\left|l\left(f^{(n)}(t)\right)\right|\right)^{k} \\
& \leqslant M_{n, k}\|f\|^{n-k}\left\|f^{(n)}\right\|^{k} .
\end{aligned}
$$

The second inequality follows since $|l|_{X^{*}}=1$. Taking the sup over $|l|_{X^{*}}$ $=1$ gives (7).

If $T(t)$ is a strongly continuous contraction semigroup with generator $A$ and $x \in \mathscr{D}\left(A^{n}\right)$, then $f(t)=T(t) x$ is $n$-times continuously differentiable and

$$
\left\|f^{(k)}\right\|=\sup _{t \geqslant 0}\left|T(t) A^{k} x\right|=\left|A^{k} x\right| .
$$

Consequently, we have the following theorem as a corollary to Theorem I.

THEOREM II. If $A$ is the generator of a strongly continuous contraction semigroup and $x$ is in the domain of $A^{n}$, some integer $n \geqslant 2$, then

$$
\left|A^{k} x\right|^{n} \leqslant M_{n, k}|x|^{n-k}\left|A^{n} x\right|^{k}
$$

for integer $k, 1 \leqslant k \leqslant n-1$.

The same approach gives the following results.

THEOREM III. Suppose $n$ is a positive integer. If $f$ is a function from $(-\infty, \infty)$ to $X$ such that $f$ has $n$ continuous derivatives and $\|f\|$ and $\left\|f^{(n)}\right\|$ are finite then

$$
\left\|f^{(k)}\right\|^{n} \leqslant C_{n, k}^{n}\|f\|^{n-k}\left\|f^{(n)}\right\|^{k}, \quad k=1,2, \ldots, n-1 .
$$

THEOREM IV. If $A$ is the generator of a strongly continuous group of isometries 
and $x$ is in the domain of $A^{n}$, some integer $n \geqslant 2$, then

$$
\left|A^{k} x\right|^{n} \leqslant C_{n, k}^{n}|x|^{n-k}\left|A^{n} x\right|^{k}
$$

for integer $k, 1 \leqslant k \leqslant n-1$.

3. Remarks. If $B$ generates a group of isometries then $B^{2}$ generates a semigroup. Since the constants on the entire line are very much smaller than those on the half-line, the converse is of interest:

For which semigroup generators $A$ is it true that $A^{1 / 2}$ generates a group of isometries?

Suppose $A^{1 / 2}$ does generate a group of isometries. Then using $n=4$ and $k=2$, Theorem IV yields

$$
\left|\left(A^{1 / 2}\right)^{2} x\right|^{4} \leqslant C_{4,2}^{4}|x|^{2}\left|\left(A^{1 / 2}\right)^{4} x\right|^{2}
$$

$x$ in the domain of $A^{2}$. Now $C_{4,2}^{4}=36 / 25$ [5]; hence, one has

$$
|A x|^{2} \leqslant \frac{6}{5}|x|\left|A^{2} x\right|
$$

however, $M_{2,1}=4$ so without using that $A^{1 / 2}$ generates a group one would have from Theorem II, $|A x|^{2} \leqslant 4|x|\left|A^{2} x\right|$.

A second question which does not seem to have been investigated at all is whether the constants of Theorem II are improved if $T$ is a holomorphic semigroup.

ACKNOWLEDGEMENT. These results extend part of the first author's dissertation written under the direction of Professor Richard Askey whose suggestions and help are gratefully acknowledged.

\section{REFERENCES}

1. A. S. Cavaretta, Jr., An elementary proof of Kolmogorov's theorem, Amer. Math. Monthly 81 (1974), 480-486.

2. M. Certain, Some theorems on semigroups and groups of operators, Ph.D. thesis, Univ. of Wisconsin, 1974.

3. Z. Ditzian, Some remarks on inequalities of Landau and Kolmogorov, Aequationes Math. 12 (1975), 145-151.

4. (1976), 80-82.

5. H. Gindler and J. Goldstein, Dissipative operator versions of some classical inequalities, J. Analyse Math. 28 (1975), 213-238.

6. J. Goldstein, On improving the constants in the Kolmogorov inequalities, Tulane Univ., 1976 (preprint).

7. E. Hille, Generalizations of Landau's inequality to linear operators, Linear Operators and Approximation, Birkhäuser, Basel, 1972, pp. 20-32.

8. J. Holbrook, A Kallman-Rota inequality for nearly Euclidean spaces, Advances in Math. 14 (1974), 335-345.

9. P. R. Kallman and G.-C. Rota, On the inequality $\left\|f^{\prime}\right\|^{2}<4\|f\|\left\|f^{\prime \prime}\right\|$, Inequalities. II. Academic Press, New York and London, 1970, pp. 187-192. 
10. A. N. Kolmogorov, On inequalities between the upper bounds of the successive derivatives of an arbitrary function on an infinite interval, Amer. Math. Soc. Transl. (1) 2 (1962), 233-243.

11. E. Landau, Einige Ungleichungen für zweimal differenziebare Funktionen, Proc. London Math. Soc. (2) 13 (1913), 43-49.

12. I. J. Schoenberg, The elementary cases of Landau's problem of inequalities between derivatives, Amer. Math. Monthly 80 (1973), 121-158.

13. I. J. Schoenberg and A. Cavaretta, Solution of Landau's problem concerning higher derivatives on the halfline, MRC T.S.R. 1060, Madison, Wis., 1970.

14. W. Trebels and U. Westphal, A note on the Landau-Kallman-Rota-Hille inequality, Linear Operators and Approximation, Birkhäuser, Basel, 1972, pp. 115-119.

Department of Mathematics, University of Wisconsin, Madison, Wisconsin 53706 